\title{
Orientation Mapping in Polycrystalline Ice Using Electron Backscatter Patterns
}

\author{
D. Iliescu,* I. Baker,* and C. P. Daghlian**
}

* Thayer School of Engineering, Dartmouth College, 8000 Cummings Hall, Hanover, NH 03755

** Center for Electron Microscopy, Dartmouth College, 244 Ramsen, Hanover, NH 03755

We recently demonstrated, that the orientations of crystals in polycrystalline ice could be determined with a higher angular and spatial resolution and more rapidly than any currently-used method by using electron back-scattered patterns (EBSPs) acquired from uncoated polycrystalline ice in a coldstage equipped scanning electron microscope (SEM) [1]. The technique was originally developed using an older Zeiss 932 thermionic emission SEM operated in high vacuum mode $\left(5 \times 10^{-4} \mathrm{~Pa}\right)$ and equipped with a custom-built cold stage cooled by cold $\mathrm{N}_{2}$ gas.

The acquisition of a new FEI XL30 field emission gun environmental SEM capable of delivering a much smaller but lower-current electron probe and the requirement that the SEM be run in environmental mode (13-17 Pa) when investigating ice necessitated a reassessment of the EBSPbased technique. We focused primarily on enhancing the quality of the EBSPs obtained with a smaller-current probe in a significantly poorer vacuum by designing a new type of cold stage (Figure 1) that allowed a series of procedural changes. The particular characteristics and advantages of the EBSP-based orientation method are discussed in [1]. We note that while the quality of the EBSPs obtained with the FEG SEM in environmental mode is evidently poorer compared to that of the patterns obtained in the Zeiss SEM in high vacuum mode, the EBSPs in the former case are still well defined and suitable for automatic indexing.

Three grains meeting at triple junction in fresh-water laboratory ice were imaged in the FEG SEM using a $15 \mathrm{KV}$ beam (Fig. 2A). The pressure in the chamber was $17 \mathrm{~Pa}$. Prior to imaging the surface of the specimen was shaved flat and was free of scratches and other macroscopic imperfections. The specimen $(20 \mathrm{~mm} \times 10 \mathrm{~mm} \times 3-4 \mathrm{~mm})$ was stored in dry ice for $3-4 \mathrm{~h}$ before being imaged. The temperature of the cold stage and removable brass holder (Fig. 1B) were maintained constant at a constant temperature of $-75^{\circ} \mathrm{C}$ (within $\pm 1^{\circ} \mathrm{C}$ ) during the entire period using a PID controller. Given the pressure in the SEM chamber $(17 \mathrm{~Pa})$, this temperature ensured that charge from the $15 \mathrm{KV}$ electron beam did not build up excessively on the specimen surface but was largely removed through sublimation. These conditions allowed both manual and automatic collection of EBSPs.

Figure 2B shows an orientation map from the area marked in Fig. 2A. To create the map the beam was translated on the specimen's surface in $10 \mu \mathrm{m}$ increments across a 69 x 54 point rectangular grid. At each point, an EBSP was automatically acquired and indexed to determine the local lattice orientation. Three indexed EBSPs corresponding to the bulk of each grain are shown in Figure 2C.

\section{References}

[1] D. Iliescu et al., Microscopy Research and Technique. 63 (2004) 183.

[2] This work was supported by the Army Research Office under contract DAAD 19-03-1-0110 and by grants from the National Science Foundation under contracts OPP-998-379 and OPP-0221120. 

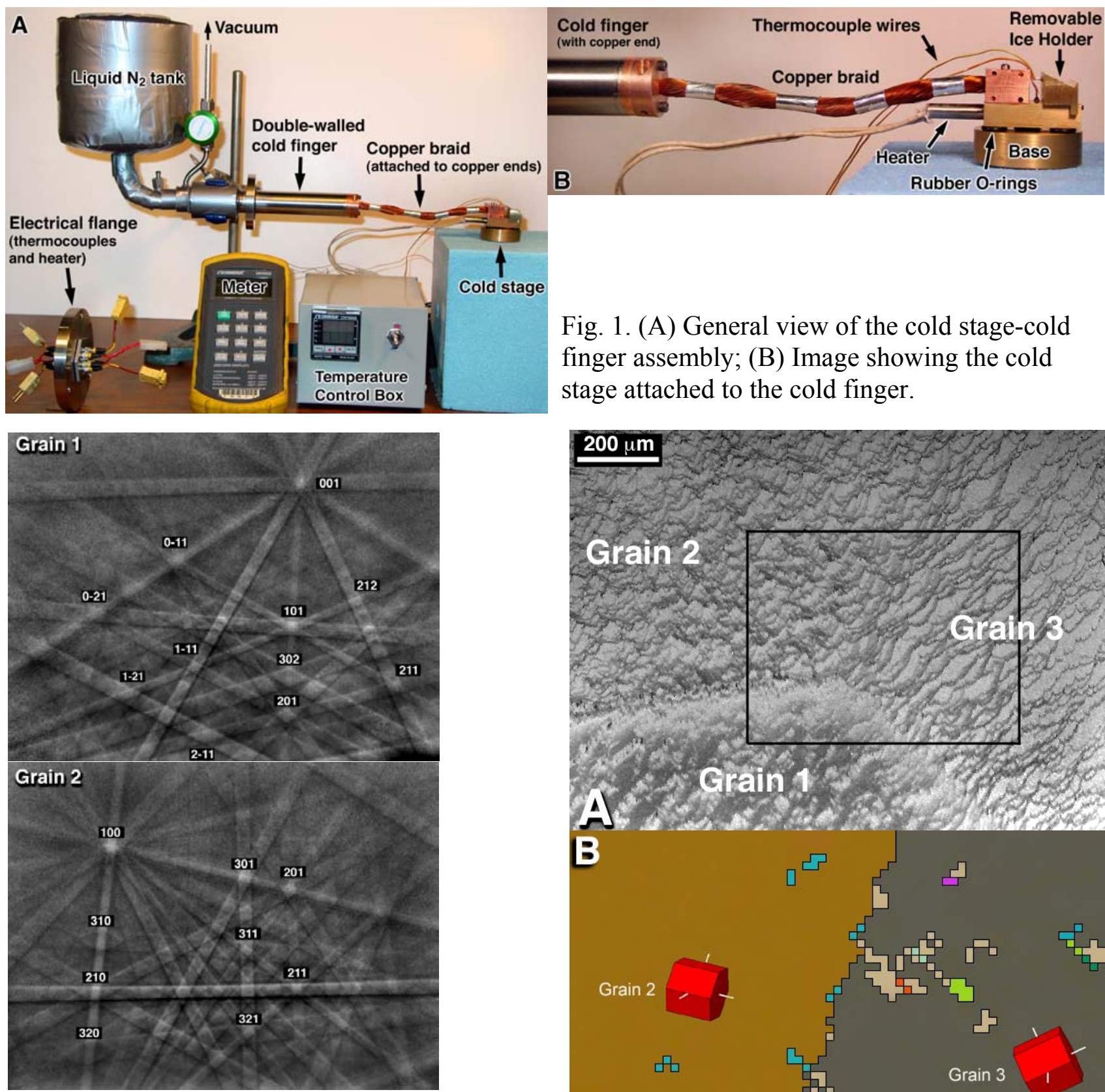

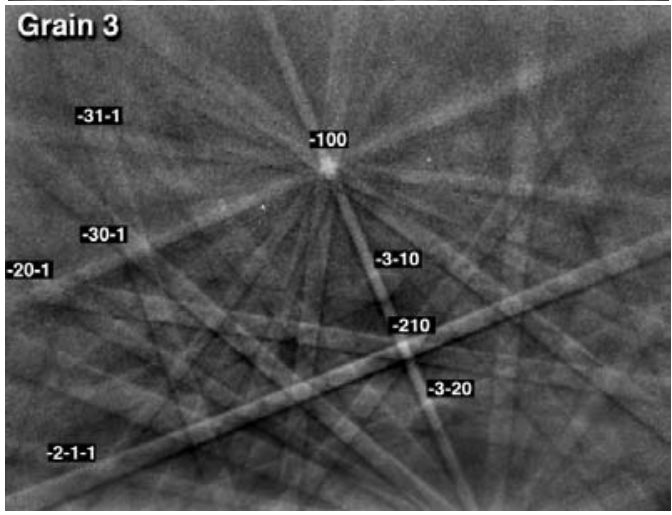

(C)

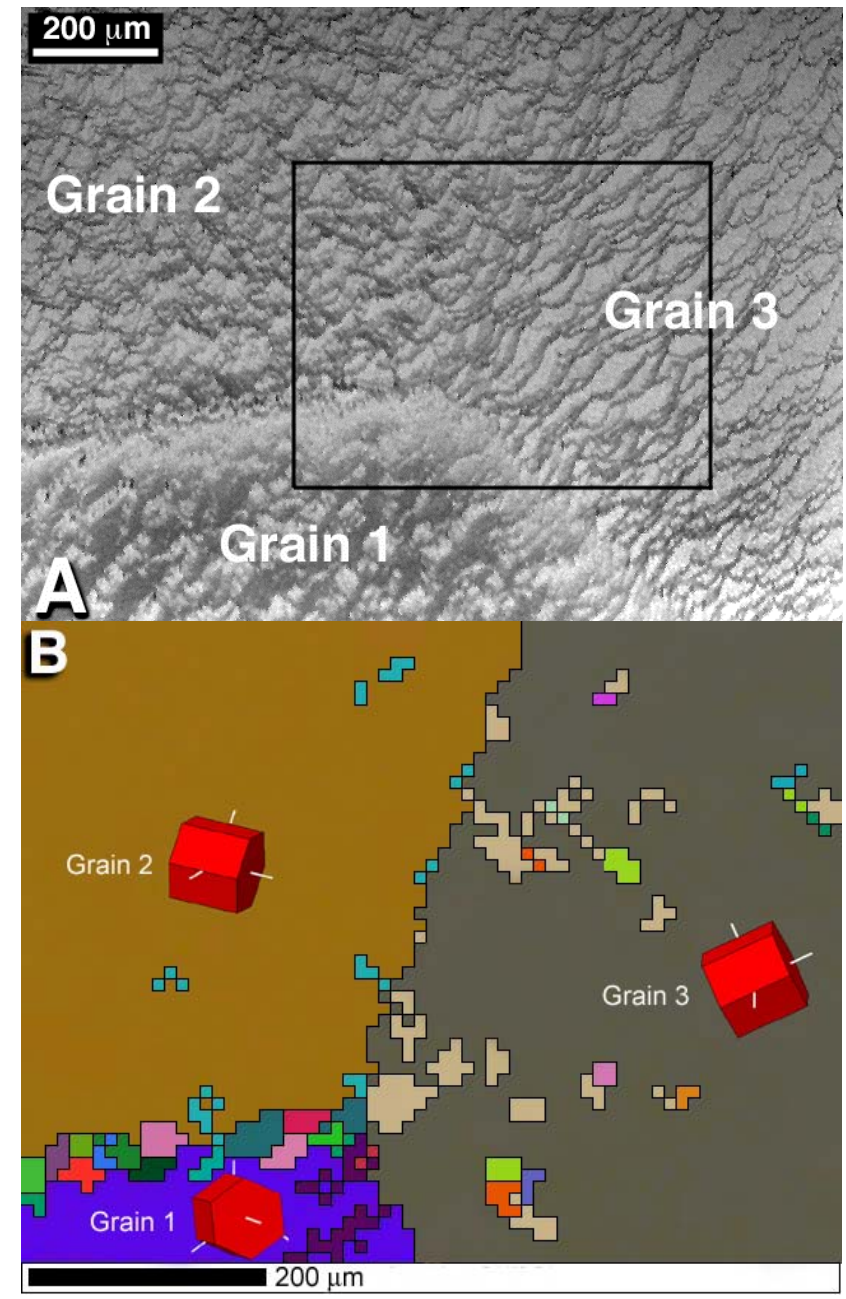

Fig. 2. (A) SEM image of a triple junction in laboratory ice; (B) orientation image from the rectangular area marked in (A). The orientation of the hexagonal unit cell in each grain is shown in insets. The orientations were computed from the EBSPs shown in (C) with respect to the specimen's surface coordinate system. 